\title{
Riociguat therapy for pulmonary hypertension: a systematic review and meta-analysis
}

\author{
Li Wang ${ }^{1,2,3,4}$, Lu Zhu ${ }^{5,6}$, Yong $\mathrm{Wu}^{1,3,4}$, Qiwei $\mathrm{Li}^{1,3,4}$, Hanmin Liu ${ }^{1,3,4}$
}

${ }^{1}$ Key Laboratory of Birth Defects and Related Diseases of Women and Children (Sichuan University), Ministry of Education, West China Second University Hospital, Sichuan University, Chengdu, China; ${ }^{2}$ Department of Pediatric Respiratory Medicine, Chengdu Women's and Children's Central Hospital, School of Medicine, University of Electronic Science and Technology of China, Chengdu, China; ${ }^{3}$ NHC Key Laboratory of Chronobiology (Sichuan University), West China Second University Hospital, Sichuan University, Chengdu, China; ${ }^{4}$ The Joint Laboratory for Lung Development and Related Diseases, West China Second University Hospital, Sichuan University, Chengdu, China; ${ }^{5}$ Department of Pediatrics, The Affiliated Hospital of Southwest Medical University, Luzhou, China; ${ }^{6}$ Sichuan Clinical Research Center for Birth Defects, Luzhou, China

Contributions: (I) Conception and design: L Wang, H Liu; (II) Administrative support: H Liu; (III) Provision of study materials or patients: L Zhu; (IV) Collection and assembly of data: L Wang, L Zhu, Y Wu, Q Li; (V) Data analysis and interpretation: L Wang, L Zhu, Y Wu, Q Li; (VI) Manuscript writing: All authors; (VII) Final approval of manuscript: All authors.

Correspondence to: Hanmin Liu. No.20, Section 3, Renmin Nan Lu, Chengdu, China. Email: liuhm@scu.edu.cn.

Background Riociguat therapy has been recommended for pulmonary arterial hypertension (PAH) and chronic thromboembolic pulmonary hypertension (CTEPH), and it might have therapeutic significance for other types of pulmonary hypertension $(\mathrm{PH})$. Our purpose was to evaluate the specific impact of riociguat on all types of $\mathrm{PH}$.

Methods: We conducted a systematic review and meta-analysis of randomized controlled trials (RCTs) comparing the safety and efficacy of riociguat treatment for $\mathrm{PH}$ through databases of the Cochrane Library, PubMed, Embase, and Web of Science from inception to the present. Duplicate publications, studies with no full text, incomplete information or inability to extract data, animal experiments and reviews, and systematic reviews were excluded. The software RevMan 5.4 was used for data synthesis.

Results: There were 8 RCTs included in our study, involving 1,606 participants. For PAH and CTEPH patients, riociguat treatment extended 6-minute walk distance (6MWD) by 39.84 meters, decreased mean pulmonary arterial pressure (PAP) by $4.20 \mathrm{mmHg}$, lowered pulmonary vascular resistance (PVR) by 218.76 dynes $/ \mathrm{sec} / \mathrm{cm}^{-5}$, cut down right atrial pressure (RAP) by $0.9 \mathrm{mmHg}$, increased cardiac index (CI) by $0.49 \mathrm{~L} / \mathrm{min} / \mathrm{m}^{2}$, improved cardiac output $(\mathrm{CO})$ by $0.89 \mathrm{~L} / \mathrm{min}$, reduced $\mathrm{N}$-terminal pro-type $\mathrm{B}$ natriuretic peptide (NT-proBNP) by $436.21 \mathrm{pg} / \mathrm{mL}$, and decreased adverse events and clinical worsening as compared with placebo. For other types of $\mathrm{PH}$ including $\mathrm{PH}$ due to left heart disease and $\mathrm{PH}$ due to lung disease, riociguat was reported as having improved CI by $0.42 \mathrm{~L} / \mathrm{min} / \mathrm{m}^{2}$ and CO was increased by $0.92 \mathrm{~L} / \mathrm{min}$ compared with placebo. Other efficacy outcomes and safety outcomes did not attain statistical difference in other types of PH.

Conclusions: For PAH and CTEPH, riociguat treatment is safe and effective, but for other types of PH, it can only improve some hemodynamic parameters.

Keywords: Riociguat; pulmonary arterial hypertension (PAH); chronic thromboembolic pulmonary hypertension (CTEPH); pulmonary hypertension $(\mathrm{PH})$; meta-analysis

Submitted Jul 26, 2021. Accepted for publication Oct 22, 2021.

doi: 10.21037/apm-21-2656

View this article at: https://dx.doi.org/10.21037/apm-21-2656 


\section{Introduction}

Pulmonary hypertension ( $\mathrm{PH}$ ) is a group of cardiopulmonary diseases characterized by increased pulmonary vascular resistance (PVR) and elevated pulmonary arterial pressure (PAP), which may lead to right ventricular (RV) hypertrophy, heart failure, and ultimately death (1). It has been reported that about $1 \%$ of the world's population have $\mathrm{PH}$, and that $10 \%$ of people over 65 years old have the disease (2). Substantial progress has been made in the development of pharmacological treatments for $\mathrm{PH}$ in the past 20 years, with 10 different targeted drugs currently available $(1,3,4)$. Nevertheless, the mortality rate of $\mathrm{PH}$ remains high, with a 5-year survival rate of less than $50 \%(5,6)$.

The definition of $\mathrm{PH}$ has recently been updated and the latest definition is that the mean arterial pressure (mPAP) is greater than $20 \mathrm{mmHg}$ at rest (7). According to the underlying causes of $\mathrm{PH}$, the $\mathrm{WHO}$ classification system divides $\mathrm{PH}$ patients into 5 groups: pulmonary arterial hypertension (group 1, PAH), $\mathrm{PH}$ due to left heart disease (group 2, PH-LHD), PH due to lung disease (group 3, LD$\mathrm{PH}$ ), chronic thromboembolic PH (group 4, CTEPH), and $\mathrm{PH}$ with unclear and/or multifactorial mechanisms (group 5) (1). Among them, LHD (group 2) is considered to be the most common cause of $\mathrm{PH}$. Up to $60 \%$ of patients with severe left ventricular (LV) systolic dysfunction and up to $70 \%$ of patients with heart failure with preserved ejection fraction could develop PH (1).

Soluble guanylate cyclase (sGC) is an effector of endogenous nitric oxide (NO). In the cardiovascular system, the combination of NO and sGC stimulates the enzyme to catalyze the conversion of guanosine 5'-triphosphate to cyclic guanosine-3',5'-monophosphate (cGMP), which could lead to several beneficial downstream physiological effects, including inhibition of vasodilation, platelet aggregation and smooth muscle cell proliferation (8). Thus, the NO-sGC-cGMP pathway has been shown to play an important role in the pathophysiologic process of $\mathrm{PH}$ (9). Riociguat is the first approved medication from the novel class of $\mathrm{sGC}$ stimulators and the only agent approved for treating both PAH and CTEPH (9-11). It could stimulate sGC directly, independent of $\mathrm{NO}$, and also sensitizes sGC to endogenous NO by stabilizing NO-sGC binding, leading to increased intracellular cGMP levels $(9,12,13)$. Thus, riociguat has antifibrotic, antiproliferative, and anti-inflammatory effects, in addition to vasodilatory properties.

A previous meta-analysis performed by Zhao et al. concluded that riociguat could improve 6-minute walk distance $(6 \mathrm{MWD})$ and hemodynamics in patients with PAH (14). However, some of the randomized controlled trials (RCTs) enrolled were actually performed on $\mathrm{PH}$ caused by systolic left ventricular dysfunction and $\mathrm{PH}$ associated with diastolic heart failure, which could not belong to PAH. Therefore, related deviations may be introduced and specific efficacy of riociguat for $\mathrm{PAH}$, as well as its safety, needs further validation.

In addition, several studies have suggested that riociguat could also improve cardiopulmonary hemodynamics of other types of PH $(15,16)$. The safety and effectiveness of riociguat in the treatment of other types of $\mathrm{PH}$ has remained undefined. Thus, our purpose was to evaluate the effectiveness and safety of riociguat treatment for all types of $\mathrm{PH}$ by conducting a systemic review and meta-analysis. We present the following article in accordance with the PRISMA reporting checklist (available at https://dx.doi. org/10.21037/apm-21-2656).

\section{Methods}

\section{Search strategy}

We searched the databases of the Cochrane Library, PubMed, Embase, and Web of Science from inception to the present with no imitations of language and publication status. The search terms were as follows: riociguat, BAY 63-2521, placebo, PAH, CTEPH, and PH. Searches were restricted to human studies. The electronic database search was supplemented by manually searching the reference list of the included articles to avoid missing any eligible studies. Studies were independently screened and selected by 2 researchers who performed quality assessment for all included studies. Any differences were resolved through discussions with a third researcher until a consensus was reached. The software RevMan 5.4 (The Cochrane Collaboration, London, UK, 2020) was used for data synthesis.

\section{Inclusion and exclusion criteria}

The inclusion criteria were as follows: (I) the study design was RCT; (II) the study population comprised patients with $\mathrm{PH}$, no matter what type of $\mathrm{PH}$; (III) intervention 
treatment included riociguat versus placebo. Studies were excluded if the research had been conducted on infants.

\section{Main outcomes}

Efficacy outcomes included the following: (I) 6MWD change; (II) cardiopulmonary hemodynamic parameters. The $6 \mathrm{MWD}$ change indicated the change in the distance walked in 6 minutes from baseline to the end of the study. Hemodynamic parameters included mPAP, PVR, right atrial pressure (RAP), cardiac index (CI), cardiac output (CO), and N-terminal pro-type B natriuretic peptide (NTproBNP).

Safety outcomes were defined as the following items: (I) adverse events; (II) clinical worsening. Adverse events included but were not limited to headache, dyspepsia, peripheral edema, nausea, dizziness, syncope diarrhea, vomiting, nasopharyngitis, hypotension, anemia, palpitations, chest pain, dyspnea, gastroesophageal reflux disease, cough, nasal congestion, tachycardia, pyrexia, fatigue, chest discomfort, flushing, gastritis, and syncope (17). Clinical worsening covered hospitalization due to $\mathrm{PH}$, initiation of new treatment for $\mathrm{PH}$, decrease in 6-min walk distance due to $\mathrm{PH}$, persistent worsening of WHO functional status due to $\mathrm{PH}$, and death (18).

\section{Bias and quality assessment}

The risk of bias for each research assessed by 2 review authors independently using the criteria outlined in the Cochrane Handbook for Systematic Reviews of Interventions (19). Bias evaluation included allocation concealment, blinding of participants and personnel, incomplete outcome data, blinding of outcome assessment, selective reporting, and other bias. Risk of bias was classified as low, high, and unclear.

\section{Data extraction}

The required data reviewed and extracted by 2 independent investigators from each study were as follows: the first author, publication year, number of patients, patient characteristics (including age, gender, etiology of $\mathrm{PH}$, body mass index, and functional class), riociguat dosage, as well as the effectiveness and safety results mentioned above. Disagreements between 2 authors were negotiated with the third investigator when necessary.

\section{Statistical analysis}

All data were synthesized and analyzed using Review Manager Version 5.4. For dichotomous data, we calculated risk ratio (RR) and the $95 \%$ confidence intervals (CI) to express the effect size. For continuous data, we estimated the mean difference or standardized mean difference and 95\% CI.

Heterogeneity across the included studies were assessed by using $\mathrm{I}^{2}$ test. When $\mathrm{I}^{2} \leq 50 \%$, heterogeneity was considered or could be neglected, and then a fixed effects model was used to merge the data. Otherwise, when $\mathrm{I}^{2}>50 \%$, significant heterogeneity was considered and could not be neglected, then sensitivity analysis was carried out. Sensitivity analysis was conducted by eliminating each included study individually, and performing a summary analysis on the remaining studies to assess whether any single included study had an excessive impact on the results of the entire meta-analysis. Subgroup analysis was conducted in accordance with the type of $\mathrm{PH}$.

\section{Results}

\section{Literature selection}

The flow chart for the selection process and detailed identification of studies is presented in Figure 1. The literature search identified 362 abstracts and titles, from which 320 records were excluded due to irrelevance or studies not conducted on humans. The remaining 42 fulltext articles were assessed for eligibility. Of these, 34 were excluded because 3 were performed on infants, 8 contained inappropriate data, and 23 trials were not RCTs. Finally, there were 8 RCTs included in the meta-analysis.

\section{Bias and quality assessment}

Results of bias risk evaluation for the enrolled RCTs are shown in Figure 2. Green represents low risk, red represents high risk, and yellow represents unclear risk and can be seen that there was no obvious bias in this study.

\section{Heterogeneity test and sensitivity analysis}

Heterogeneity was observed for the meta-analysis of CI and clinical worsening, thus we did sensitivity analysis. None of the studies had a serious influence on the results of the meta-analysis, indicating that the results of the remaining 
Identification of studies via databases and registers

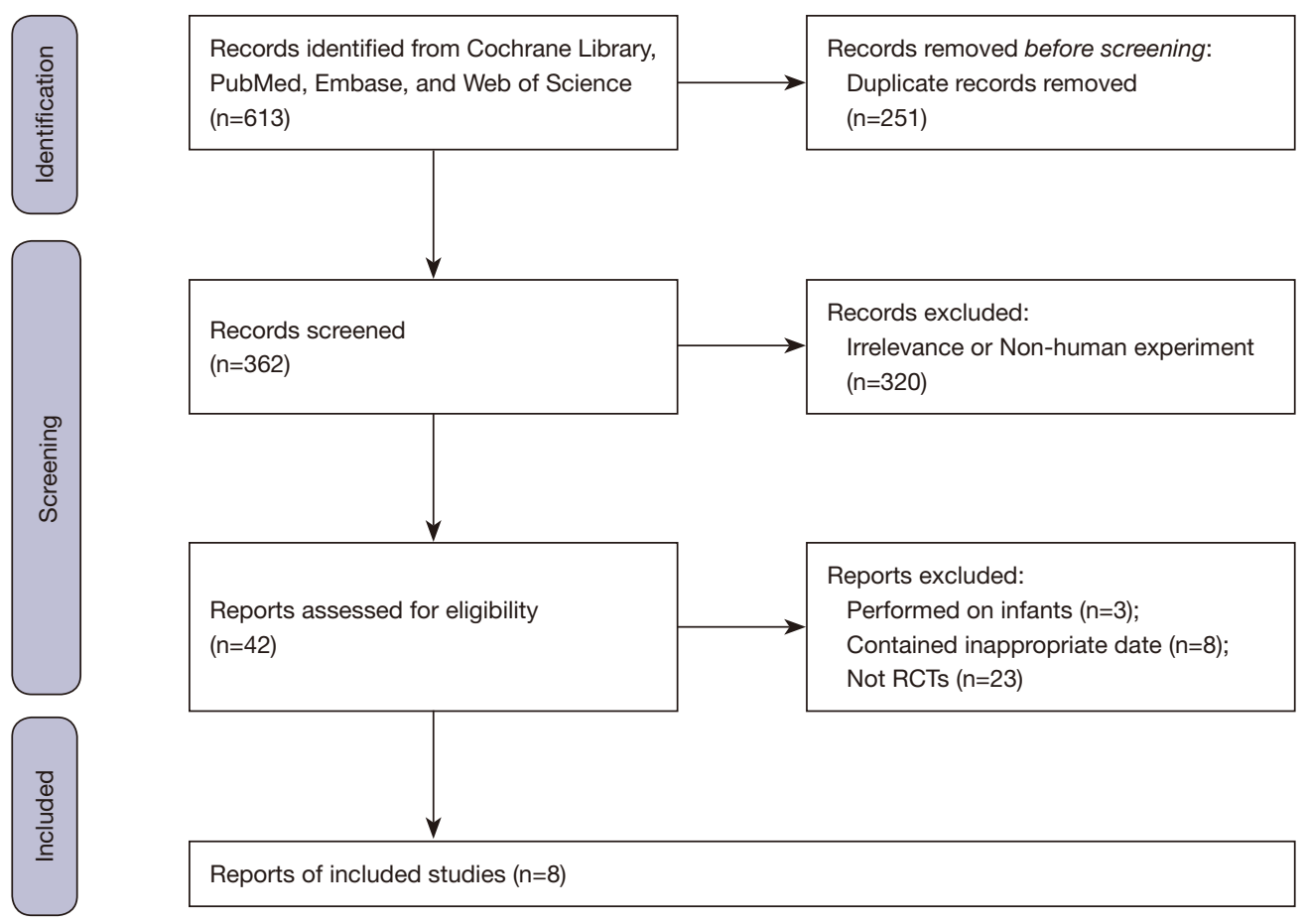

Figure 1 Flow chart of the literature search. RCT, randomized controlled trial.

studies were reliable.

\section{Characteristics of the studies}

A total of 5 studies had been conducted on PAH and CTEPH patients $(n=1,230)$, of which 810 were on a riociguat treated group and 420 on a placebo group (18,20-23). One study included inoperable CTEPH and persistent/recurrent $\mathrm{PH}$ following pulmonary endarterectomy (PEA) and their independent control group (23), which we analyzed separately. Another study focused on patients with PAH who were treatment naive or pretreated with endothelin receptor antagonists (ERAs) or non-intravenous prostanoids (22), which we also included and analyzed separately. There were 2 studies that had been conducted by Ghofrani with different colleagues in 2013, where PH and CTEPH were discussed respectively $(18,20)$. Another 3 studies were carried out on other types of $\mathrm{PH}$ $(\mathrm{n}=376)$, with 202 patients in the riociguat group and 174 in the placebo group $(15,16,24)$. The 3 studies included $\mathrm{PH}$ associated with diastolic heart failure, $\mathrm{PH}$ caused by systolic left ventricular dysfunction, and idiopathic interstitial pneumonia-associated $\mathrm{PH}$, which belonged to group 2, group 2, and group 3 of $\mathrm{PH}$, separately. The baseline characteristics of 8 eligible RCTs in the meta-analysis are summarized in Table 1. Participants in riociguat group and placebo group showed similar baseline characteristics. The dose of riociguat varied from $1.5 \mathrm{mg}$ daily to $2.5 \mathrm{mg}$ 3 times daily and follow-up period lasted between 12 and 16 weeks in 7 trials. There was 1 study that investigated single oral doses of $2 \mathrm{mg}$ and hemodynamic parameters were recorded at regular intervals up to $6 \mathrm{~h}$ after intake of the study drug (16).

\section{Efficacy assessment}

\section{Exercise capacity}

Exercise capacity was evaluated with $6 \mathrm{MWD}$ changes in 5 articles, of 3 which were implemented in $\mathrm{PAH}$ and CTEPH, and 2 in other types of PH subset (Figure 3). For $\mathrm{PAH}$ and CTEPH, participants treated with riociguat could walk 39.84 meters further than those receiving placebo $(\mathrm{P}<0.00001)$, while in other types of $\mathrm{PH}$, the 6MWD change between riociguat and placebo group did not reach 

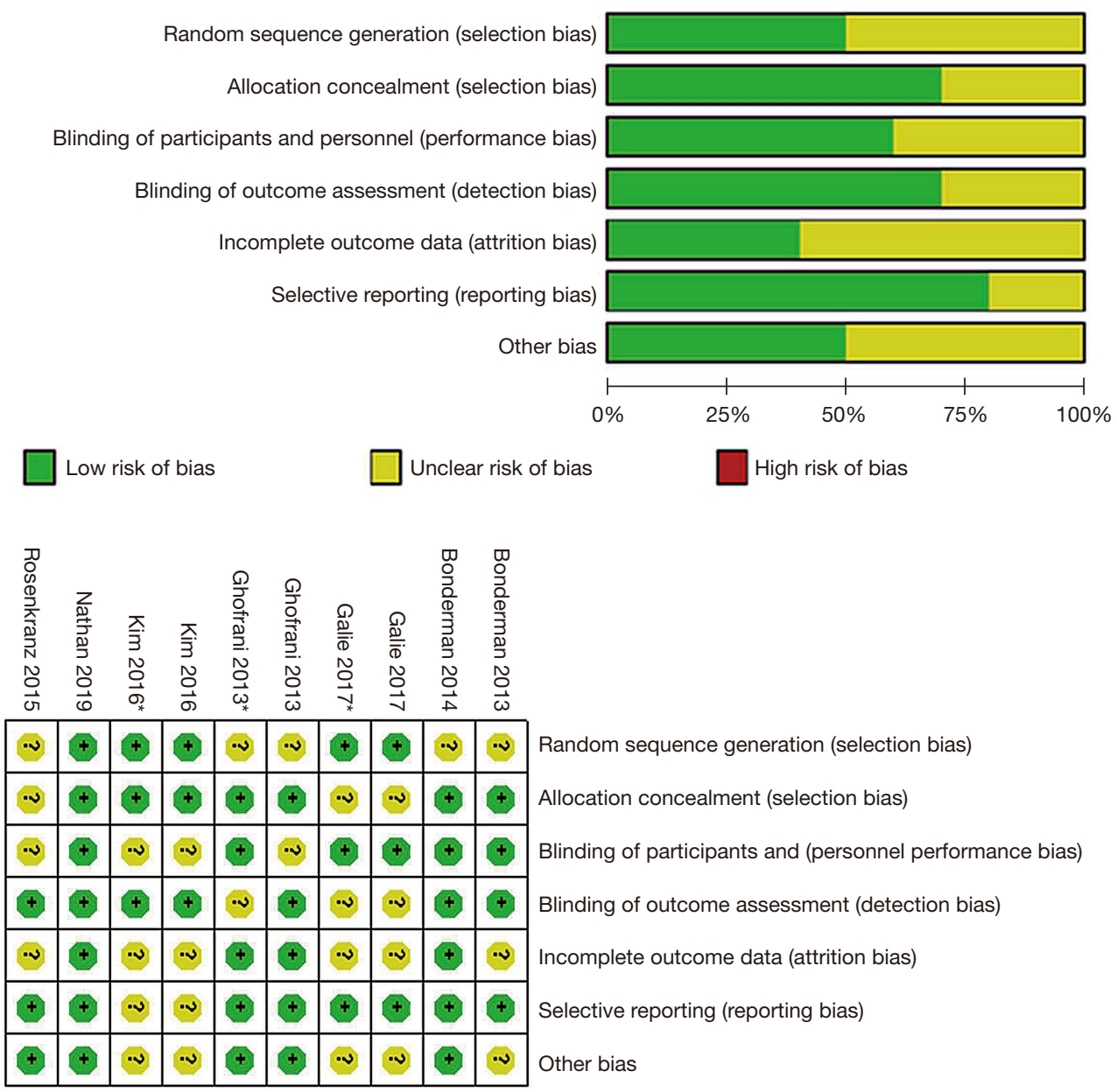

Figure 2 Risk of bias graph and risk of bias summary.

statistical significance $(\mathrm{P}=0.16)$. These results were stable in sensitivity analysis. Funnel plots indicated no obvious publication bias.

\section{Cardiopulmonary hemodynamics}

All of the included studies reported parameters of mPAP, PVR, and RAP. Riociguat therapy reduced mPAP by 4.20 $\mathrm{mmHg}$ in PAH and CTEPH participants compared with placebo $(\mathrm{P}<0.00001$; Figure 4$)$, and $\mathrm{PVR}$ was reduced by 218.76 dynes $/ \mathrm{se} / \mathrm{cm}^{-5}$ in the riociguat group than that in placebo group $(\mathrm{P}<0.00001$; Figure 5$)$. Furthermore, RAP was cut down by $0.9 \mathrm{mmHg}$ in $\mathrm{PAH}$ and CTEPH participants compared with placebo ( $\mathrm{P}=0.003$; Figure 6). However, in other types of $\mathrm{PH}, \mathrm{mPAP}, \mathrm{RVP}$, and RAP did not reach significant difference between groups (Figures 4-6).

For PAH and CTEPH, 3 studies reported CI, CO, and NT-proBNP. Compared with placebo, riociguat improved
CI by $0.49 \mathrm{~L} / \mathrm{min} / \mathrm{m}^{2}(\mathrm{P}<0.00001$; Figure 7), increased CO by $0.89 \mathrm{~L} / \mathrm{min}(\mathrm{P}<0.00001$; Figure 8$)$, and reduced NTproBNP by $436.21 \mathrm{pg} / \mathrm{mL}(\mathrm{P}=0.0003$; Figure 9). For other types of $\mathrm{PH}, 3$ studies reported CI, 1 study recorded $\mathrm{CO}$ and, another 1 collected NT-proBNP. The CI was increased by $0.42 \mathrm{~L} / \mathrm{min} / \mathrm{m}^{2}(\mathrm{P}<0.00001$; Figure 7$)$, and $\mathrm{CO}$ was increased by $0.92 \mathrm{~L} / \mathrm{min}$ compared with placebo reported $(\mathrm{P}=0.001$; Figure 8). Meanwhile, NT-proBNP did not show a significant difference between groups $(\mathrm{P}=0.56$; Figure 9)

\section{Safety assessment}

There were 8 studies that reported adverse events after riociguat treatment, 5 of which were performed in $\mathrm{PAH}$ and CTEPH, and 3 in other types of PH subset. Compared with placebo, riociguat treatment could not increase the rate of adverse events in PAH and CTEPH $(\mathrm{P}=0.06$; 


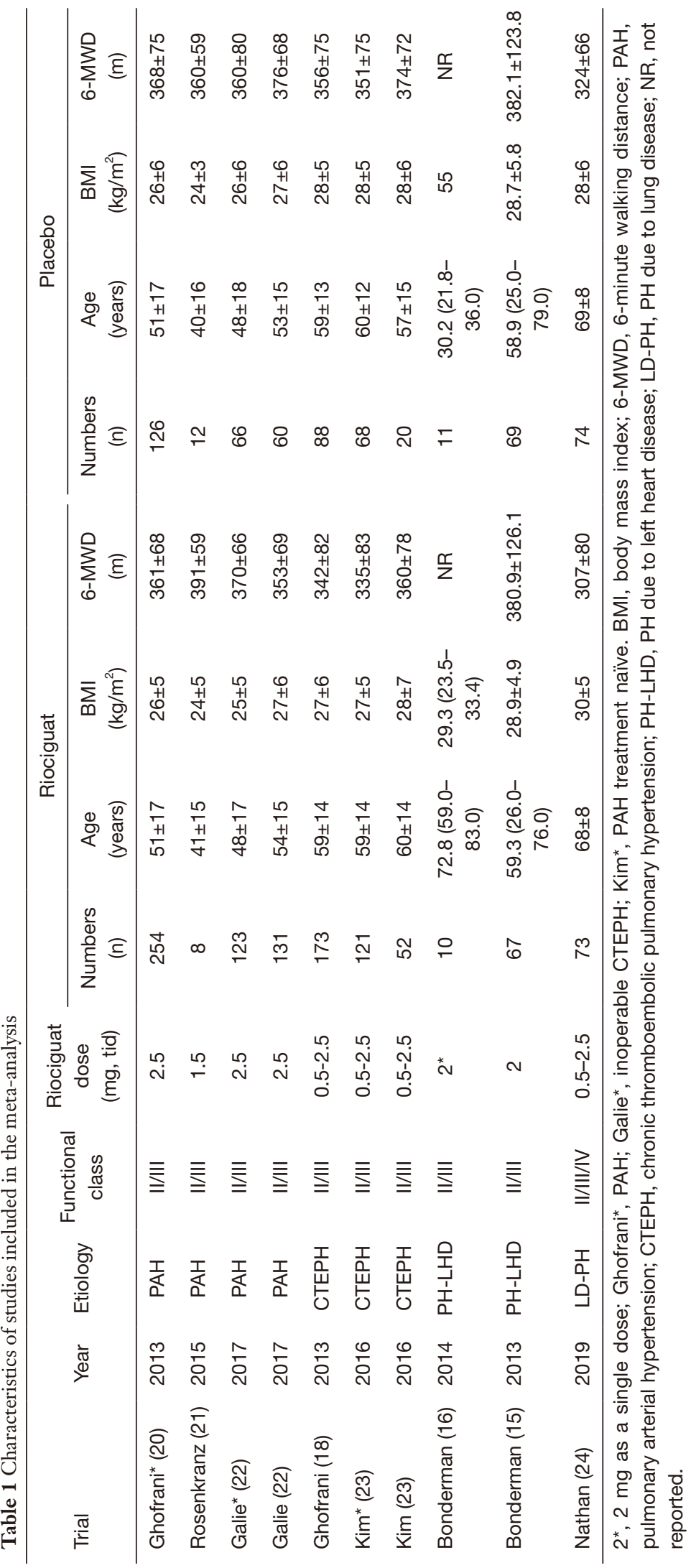




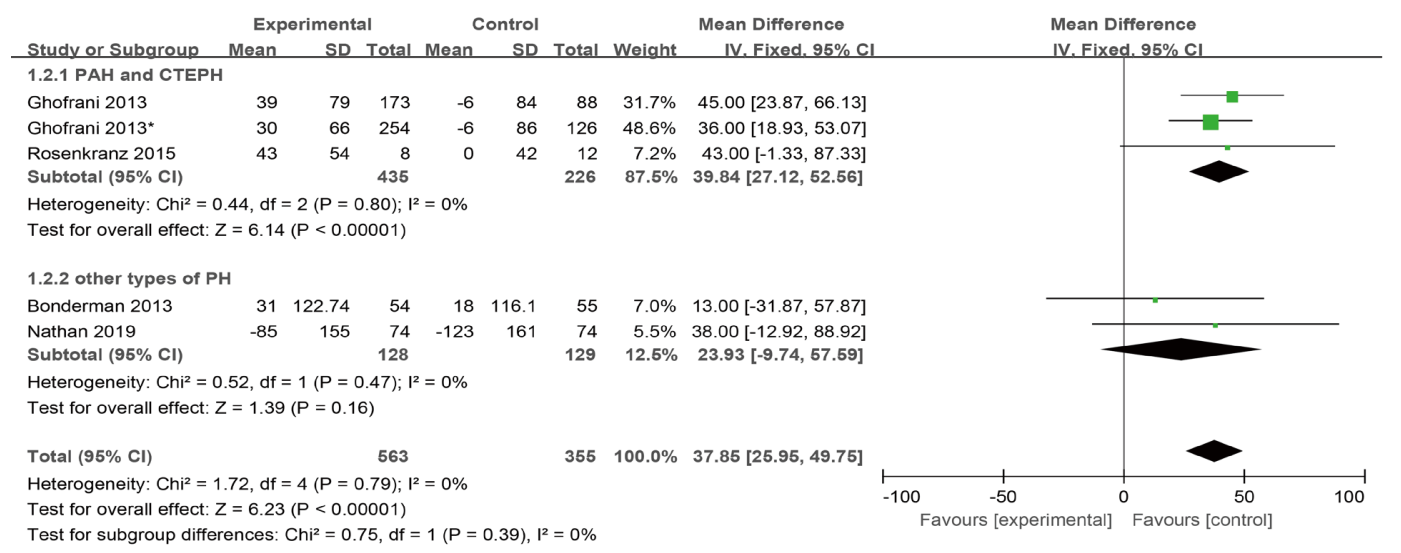

Figure 3 Meta-analysis results of riociguat on change in $6 \mathrm{MWD}$. 6MWD, 6-minute walk distance.

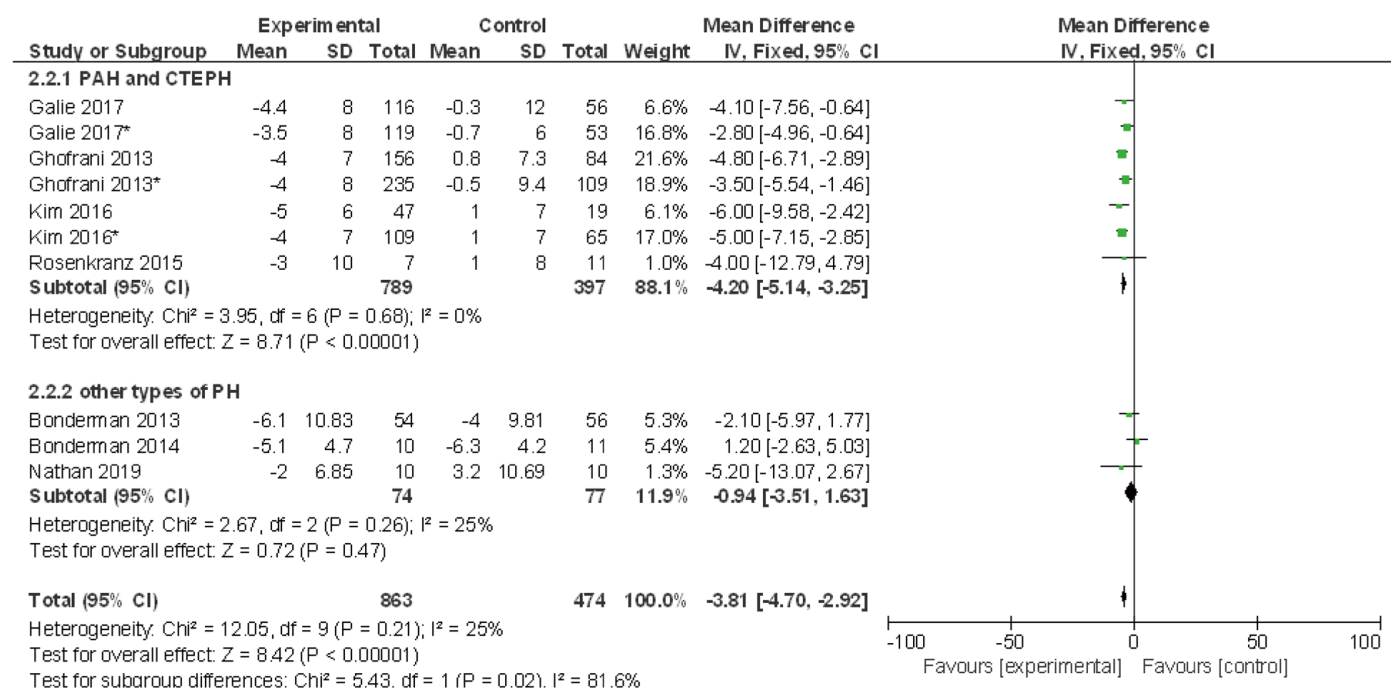

Figure 4 Meta-analysis results of riociguat on change in mPAP. mPAP, mean pulmonary arterial pressure.

Figure 10), but in other types of $\mathrm{PH}$, riociguat treatment was associated with approximately 1.15 -fold higher than the rate of adverse events $(\mathrm{P}=0.006$; Figure 10). There were 3 studies that recorded outcomes of clinical worsening of $\mathrm{PAH}$ and CTEPH, with no statistical difference between groups $(\mathrm{P}=0.22$; Figure 11), and no data were available for other types of PH. These results were stable in sensitivity analysis.

\section{Discussion}

This meta-analysis of riociguat therapy for PH revealed that riociguat was relatively safe and could improve clinical outcome of patients in PAH and CTEPH, whereas it only relieved certain hemodynamics of other types of $\mathrm{PH}$.

Change in $6 \mathrm{MWD}$ was reported to have an important relationship with functional status, hemodynamic variables, and survival and served as the most frequently used indicator of exercise capacity (25-27). The RAP, CI, CO are the most important indicators of right ventricle (RV) function and prognosis, among which RV function plays as a key and determinant role of exercise capacity and outcome in $\mathrm{PH}$ patients $(28,29)$. In addition, NT-proBNP acts as a biochemical marker in patients with $\mathrm{PH}$, which is associated with myocardial dysfunction and could provide reliable prognostic information at the time of diagnosis and during follow-up assessments (30-32). For PAH and CTEPH patients, our study revealed that riociguat 


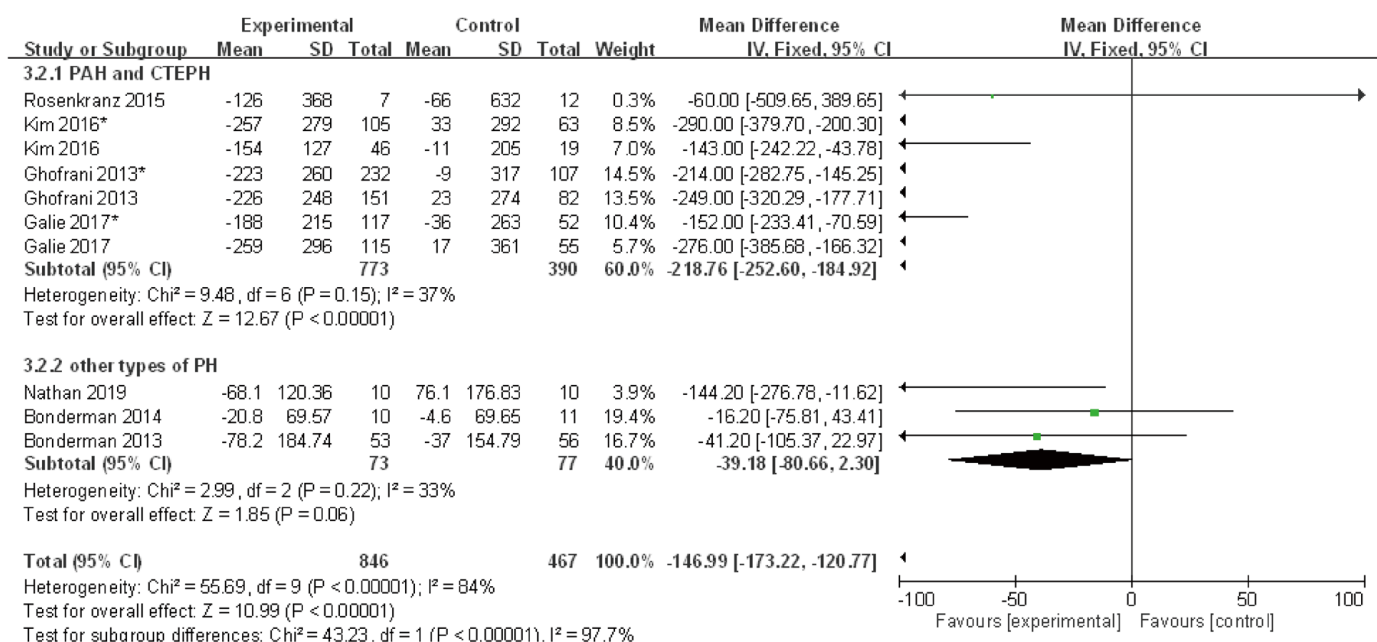

Figure 5 Meta-analysis results of riociguat on change in PVR. PVR, pulmonary vascular resistance.

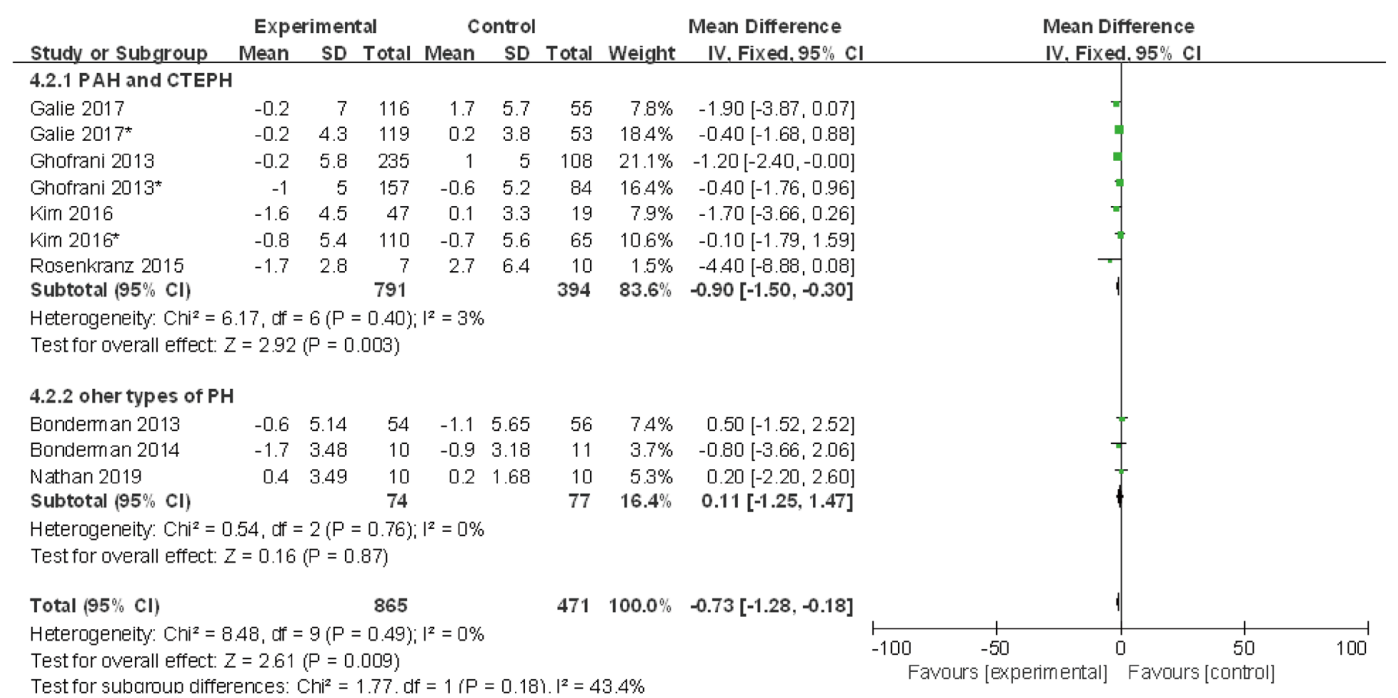

Figure 6 Meta-analysis results of riociguat on change in RAP. RAP, right atrial pressure.

significantly enhanced cardiopulmonary hemodynamic parameters, which include mPAP, PVR, RAP, CI, CO, and NT-proBNT, and increased exercise capacity compared with placebo. Furthermore, riociguat also reduced the rate of clinical worsening and adverse events. For other types of $\mathrm{PH}$, we concluded that only $\mathrm{CI}$ and $\mathrm{CO}$ were ameliorated after riociguat treatment.

The similarity in hemodynamics and pathophysiology between CTEPH and PAH provides the rationale for clinical use of the same therapy (33). Thus, the differences in efficacy of riociguat between PAH and CTEPH with the other types of $\mathrm{PH}$ might be owing to their distinct nature. Independent of etiology, pulmonary vascular remodeling is one of the main characteristics of all forms of PH (34). In $\mathrm{PAH}$, vascular remodeling was mainly concentrated in the middle and small pulmonary arteries, while PH-LHD are characterized predominantly by venous remodeling (35). In addition, different types of $\mathrm{PH}$ have different treatment goals. For PH-LHD, the main goal of treatment must be to improve the underlying diseases before considering specific treatment measures of $\mathrm{PH}$, including repair of valvular heart disease when indicated and aggressive therapy for 


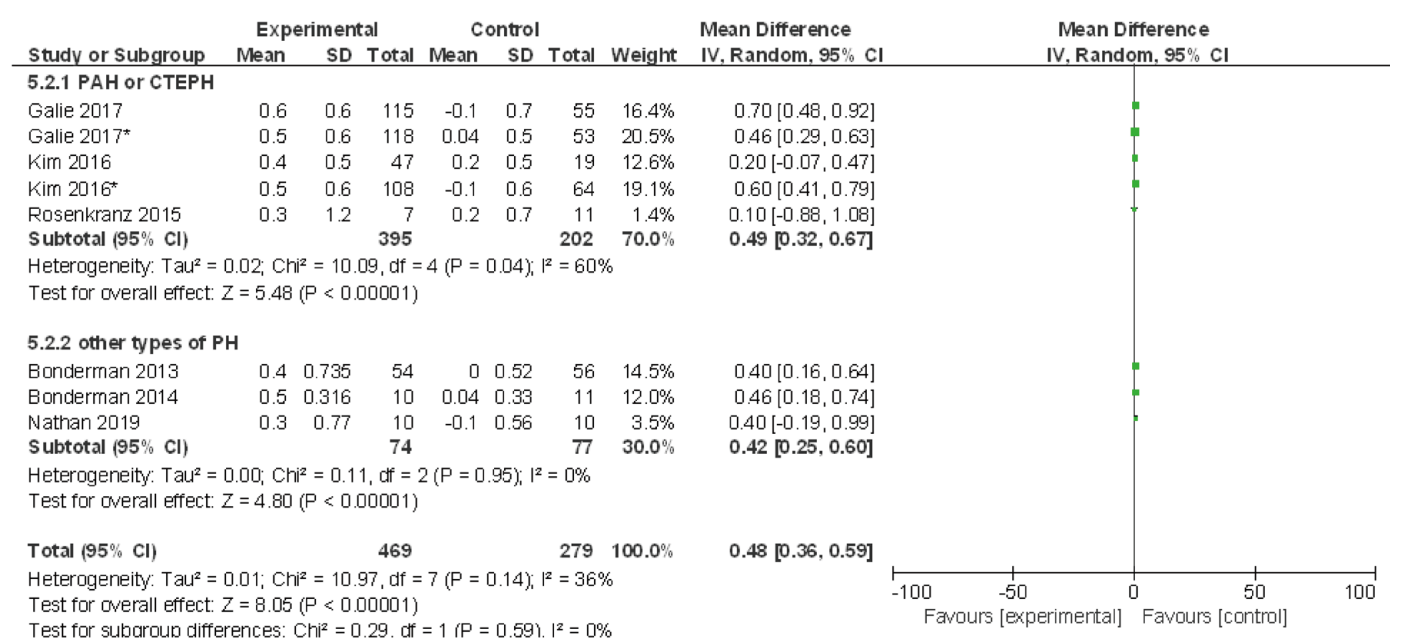

Figure 7 Meta-analysis results of riociguat on change in CI. CI, cardiac index.

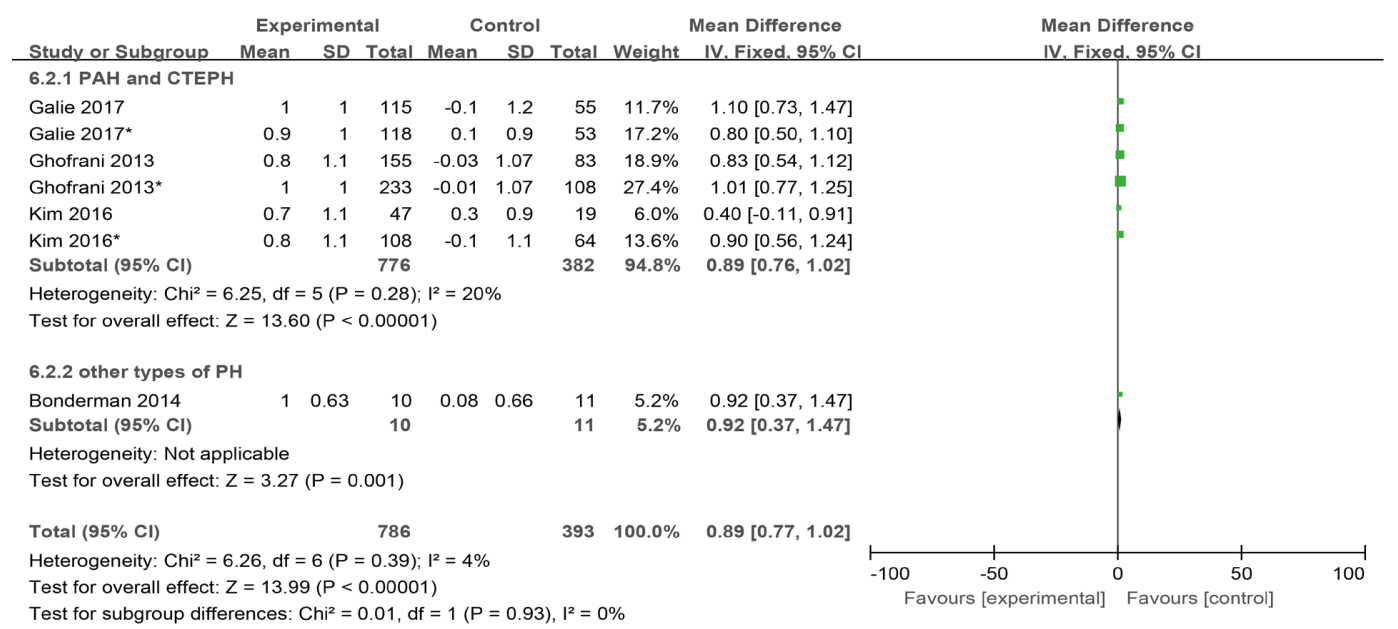

Figure 8 Meta-analysis results of riociguat on change in CO. CO, cardiac output.

heart failure with reduced systolic function (36). Therefore, we suspected that the unique pathophysiology of different subtypes of $\mathrm{PH}$ leads to the heterogeneity between them regarding exercise capacity and cardiopulmonary hemodynamics changes.

\section{Limitations}

Several limitations existed in our study. Firstly, due to limitation of related research and available data, we did not conduct subgroup analysis based on different etiology of $\mathrm{PH}$ in different groups. Secondly, we did not analyze the impact of riociguat on patients with different functional class. Thirdly, there were only 3 related RCTs on other types of PH. Small sample size might be a problem and more RCTs with sufficient sample sizes enrolled are needed to expand and validate our findings.

\section{Conclusions}

Our meta-analysis showed that riociguat effectively improves exercise capacity and cardiopulmonary hemodynamics of PAH and CTEPH patients, and it is relatively safe and well tolerated. For other types of $\mathrm{PH}$, apart from amelioration of certain hemodynamic parameters, riociguat could not improve symptoms or 


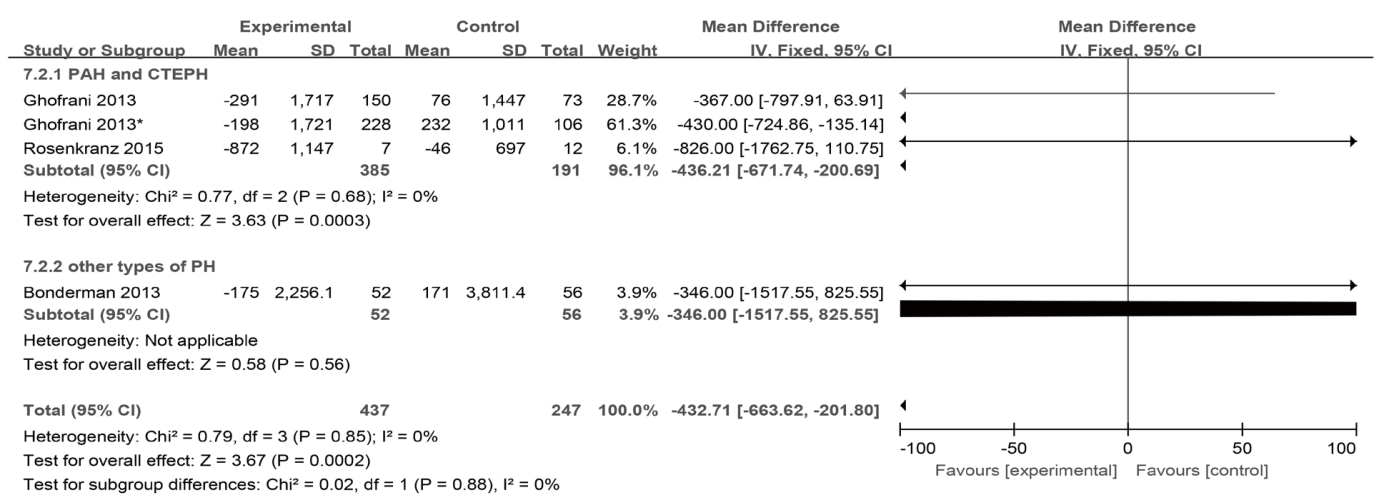

Figure 9 Meta-analysis results of riociguat on change in NT-proBNP. NT-proBNP, N-terminal pro-type B natriuretic peptide.

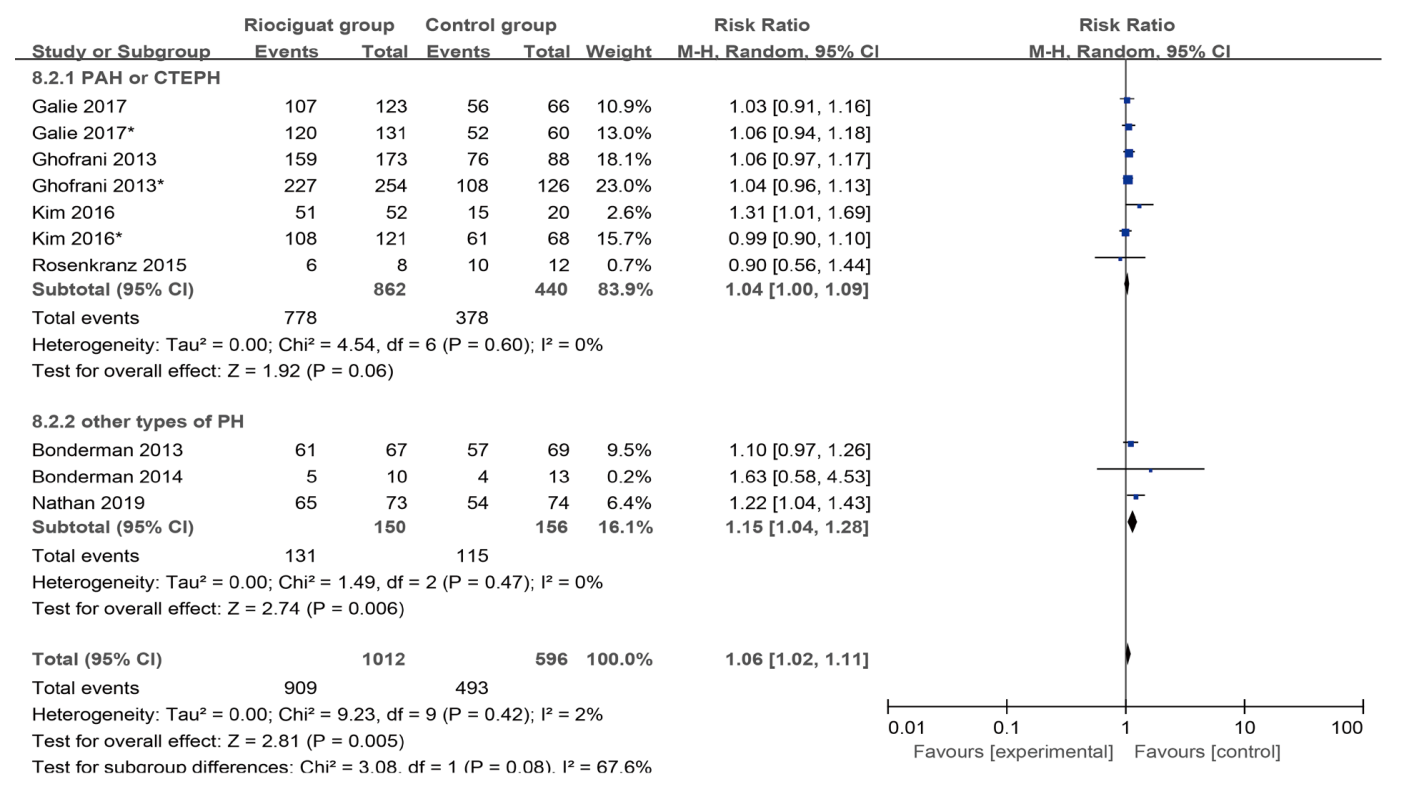

Figure 10 Meta-analysis results of riociguat on adverse events.

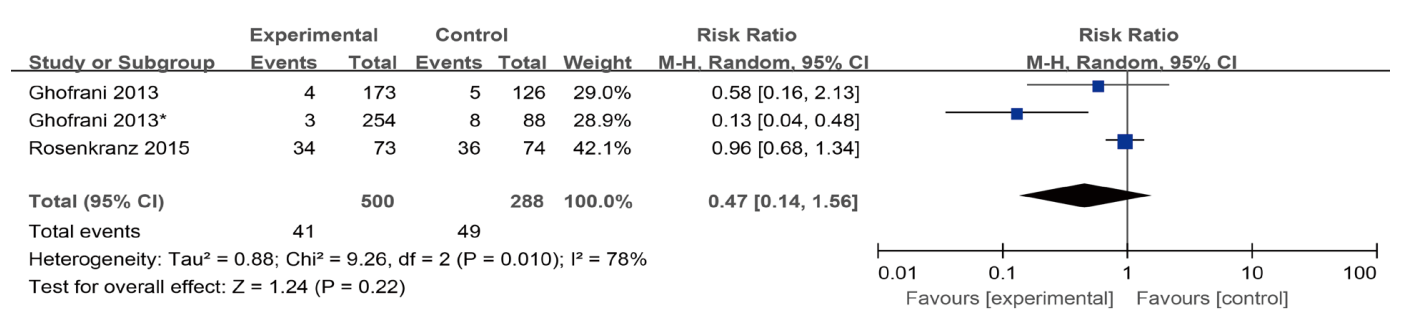

Figure 11 Meta-analysis results of riociguat on clinical worsening. 
prevent deterioration.

\section{Acknowledgments}

Funding: This research was supported by Sichuan Science and Technology Program (2019YFS0037).

\section{Footnote}

Reporting Checklist: The authors have completed the PRISMA reporting checklist. Available at https://dx.doi. org/10.21037/apm-21-2656

Conflicts of Interest: All authors have completed the ICMJE uniform disclosure form (available at https://dx.doi. org/10.21037/apm-21-2656). The authors have no conflicts of interest to declare.

Ethical Statement: The authors are accountable for all aspects of the work in ensuring that questions related to the accuracy or integrity of any part of the work are appropriately investigated and resolved.

Open Access Statement: This is an Open Access article distributed in accordance with the Creative Commons Attribution-NonCommercial-NoDerivs 4.0 International License (CC BY-NC-ND 4.0), which permits the noncommercial replication and distribution of the article with the strict proviso that no changes or edits are made and the original work is properly cited (including links to both the formal publication through the relevant DOI and the license). See: https://creativecommons.org/licenses/by-nc-nd/4.0/.

\section{References}

1. Galiè N, Humbert M, Vachiery JL, et al. 2015 ESC/ERS Guidelines for the diagnosis and treatment of pulmonary hypertension: The Joint Task Force for the Diagnosis and Treatment of Pulmonary Hypertension of the European Society of Cardiology (ESC) and the European Respiratory Society (ERS): Endorsed by: Association for European Paediatric and Congenital Cardiology (AEPC), International Society for Heart and Lung Transplantation (ISHLT). Eur Heart J 2016;37:67-119.

2. Hoeper MM, Humbert M, Souza R, et al. A global view of pulmonary hypertension. Lancet Respir Med 2016;4:306-22.

3. McLaughlin VV, Archer SL, Badesch DB, et al. ACCF/
AHA 2009 expert consensus document on pulmonary hypertension a report of the American College of Cardiology Foundation Task Force on Expert Consensus Documents and the American Heart Association developed in collaboration with the American College of Chest Physicians; American Thoracic Society, Inc.; and the Pulmonary Hypertension Association. J Am Coll Cardiol 2009;53:1573-619.

4. Vazquez ZGS, Klinger JR. Guidelines for the Treatment of Pulmonary Arterial Hypertension. Lung 2020;198:581-96.

5. Koudstaal T, Boomars KA, Kool M. Pulmonary Arterial Hypertension and Chronic Thromboembolic Pulmonary Hypertension: An Immunological Perspective. J Clin Med 2020;9:561.

6. Jing ZC, Xu XQ, Han ZY, et al. Registry and survival study in chinese patients with idiopathic and familial pulmonary arterial hypertension. Chest 2007;132:373-9.

7. Simonneau G, Montani D, Celermajer DS, et al. Haemodynamic definitions and updated clinical classification of pulmonary hypertension. Eur Respir J 2019;53:1801913.

8. Lian TY, Jiang X, Jing ZC. Riociguat: a soluble guanylate cyclase stimulator for the treatment of pulmonary hypertension. Drug Des Devel Ther 2017;11:1195-207.

9. Brockunier L, Stelmach J, Guo J, et al. Soluble guanylate cyclase stimulators for the treatment of hypertension: Discovery of MK-2947. Bioorganic \& medicinal chemistry letters 2020;30:127574.

10. Makowski CT, Rissmiller RW, Bullington WM. Riociguat: a novel new drug for treatment of pulmonary hypertension. Pharmacotherapy 2015;35:502-19.

11. Klinger JR, Chakinala MM, Langleben D, et al. Riociguat: Clinical research and evolving role in therapy. Br J Clin Pharmacol 2021;87:2645-62.

12. Stasch JP, Evgenov OV. Soluble guanylate cyclase stimulators in pulmonary hypertension. Handb Exp Pharmacol 2013;218:279-313.

13. Ghofrani HA, Humbert M, Langleben D, et al. Riociguat: Mode of Action and Clinical Development in Pulmonary Hypertension. Chest 2017;151:468-80.

14. Zhao R, Jiang Y. Influence of riociguat treatment on pulmonary arterial hypertension : A meta-analysis of randomized controlled trials. Herz 2019;44:637-43.

15. Bonderman D, Ghio S, Felix SB, et al. Riociguat for patients with pulmonary hypertension caused by systolic left ventricular dysfunction: a phase IIb doubleblind, randomized, placebo-controlled, dose-ranging 
hemodynamic study. Circulation 2013;128:502-11.

16. Bonderman D, Pretsch I, Steringer-Mascherbauer R, et al. Acute hemodynamic effects of riociguat in patients with pulmonary hypertension associated with diastolic heart failure (DILATE-1): a randomized, double-blind, placebocontrolled, single-dose study. Chest 2014;146:1274-85.

17. Liu QQ, Jing ZC. The limits of oral therapy in pulmonary arterial hypertension management. Ther Clin Risk Manag 2015;11:1731-41.

18. Ghofrani HA, D'Armini AM, Grimminger F, et al. Riociguat for the treatment of chronic thromboembolic pulmonary hypertension. N Engl J Med 2013;369:319-29.

19. Higgins J, Green S. Cochrane Handbook for Systematic Reviews of Interventions. 2019.

20. Ghofrani HA, Galiè N, Grimminger F, et al. Riociguat for the treatment of pulmonary arterial hypertension. N Engl J Med 2013;369:330-40.

21. Rosenkranz S, Ghofrani HA, Beghetti M, et al. Riociguat for pulmonary arterial hypertension associated with congenital heart disease. Heart 2015;101:1792-9.

22. Galiè N, Grimminger F, Grünig E, et al. Comparison of hemodynamic parameters in treatment-naïve and pretreated patients with pulmonary arterial hypertension in the randomized phase III PATENT-1 study. J Heart Lung Transplant 2017;36:509-19.

23. Kim NH, D'Armini AM, Grimminger F, et al. Haemodynamic effects of riociguat in inoperable/recurrent chronic thromboembolic pulmonary hypertension. Heart 2017;103:599-606.

24. Nathan SD, Behr J, Collard HR, et al. Riociguat for idiopathic interstitial pneumonia-associated pulmonary hypertension (RISE-IIP): a randomised, placebo-controlled phase 2b study. Lancet Respir Med 2019;7:780-90.

25. Fritz JS, Blair C, Oudiz RJ, et al. Baseline and follow-up 6-min walk distance and brain natriuretic peptide predict 2-year mortality in pulmonary arterial hypertension. Chest 2013;143:315-23.

26. Gabler NB, French B, Strom BL, et al. Validation of 6-minute walk distance as a surrogate end point in pulmonary arterial hypertension trials. Circulation

Cite this article as: Wang L, Zhu L, Wu Y, Li Q, Liu H. Riociguat therapy for pulmonary hypertension: a systematic review and meta-analysis. Ann Palliat Med 2021;10(10):1111711128. doi: 10.21037/apm-21-2656
2012;126:349-56.

27. Savarese G, Paolillo S, Costanzo P, et al. Do changes of 6-minute walk distance predict clinical events in patients with pulmonary arterial hypertension? A metaanalysis of 22 randomized trials. J Am Coll Cardiol 2012;60:1192-201.

28. Sitbon O, McLaughlin VV, Badesch DB, et al. Survival in patients with class III idiopathic pulmonary arterial hypertension treated with first line oral bosentan compared with an historical cohort of patients started on intravenous epoprostenol. Thorax 2005;60:1025-30.

29. McLaughlin VV. Survival in patients with pulmonary arterial hypertension treated with first-line bosentan. Eur J Clin Invest 2006;36 Suppl 3:10-5.

30. Souza R, Jardim C, Julio Cesar Fernandes C, et al. NTproBNP as a tool to stratify disease severity in pulmonary arterial hypertension. Respir Med 2007;101:69-75.

31. Leuchte HH, El Nounou M, Tuerpe JC, et al. N-terminal pro-brain natriuretic peptide and renal insufficiency as predictors of mortality in pulmonary hypertension. Chest 2007;131:402-9.

32. Souza R, Jardim C, Carvalho C, et al. The role of NT-proBNP as a prognostic marker in pulmonary hypertension. Chest 2006;130:1627-8.

33. Tian Z, Jiang X, Jing ZC. How should a physician approach the pharmacological management of chronic thromboembolic pulmonary hypertension? Expert Opin Pharmacother 2021;22:557-63.

34. Humbert M, Guignabert C, Bonnet S, et al. Pathology and pathobiology of pulmonary hypertension: state of the art and research perspectives. Eur Respir J 2019;53:1801887.

35. Tuder RM, Archer SL, Dorfmüller P, et al. Relevant issues in the pathology and pathobiology of pulmonary hypertension. J Am Coll Cardiol 2013;62:D4-12.

36. Charalampopoulos A, Lewis R, Hickey $\mathrm{P}$, et al. Pathophysiology and Diagnosis of Pulmonary Hypertension Due to Left Heart Disease. Front Med (Lausanne) 2018;5:174.

(English Language Editor: J. Jones) 